\title{
Hybrid Photo-Detectors for the Hyper-Kamiokande Project
}

\section{Yasuhiro NISHIMURA ${ }^{* \dagger}$}

The University of Tokyo

E-mail: ynisidicrr.u-tokyo.ac.jp

\begin{abstract}
Hyper-Kamiokande is a water Cherenkov neutrino detector planned for the near future. Its fiducial volume is 25 times larger than that of Super-Kamiokande. Several photo-detector (PD) candidates, such as a 20-inch Hybrid Photo-Detector (HPD) with an avalanche diode are now being developed. Plans to test a smaller, 8-inch, version of this HPD in a 200-ton water Cherenkov detector loaded with Gadolinium are underway. Pre-calibration and performance evaluation of these 8-inch HPDs are being performed prior to their installation in the tank. The plan for HPD test inside of the 200-ton detector is introduced.
\end{abstract}

International Workshop on New Photon-detectors

June 13-15, 2012

LAL Orsay, France

* Speaker.

${ }^{\dagger}$ On behalf of the Hyper-K working group 


\section{Introduction}

\subsection{Hyper-Kamiokande project}

The Hyper-Kamiokande (Hyper-K) project is proposed as a next generation underground water

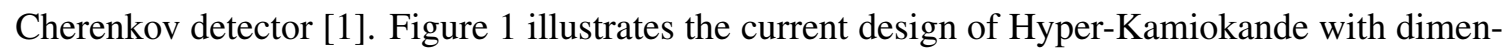
sions $48(\mathrm{~W}) \times 54(\mathrm{H}) \times 250(\mathrm{~L}) \mathrm{m}^{3}$. Its total (fiducial) volume is $1.0(0.56)$ million metric tons, which is 20 (25) times larger than that of Super-Kamiokande [[]].

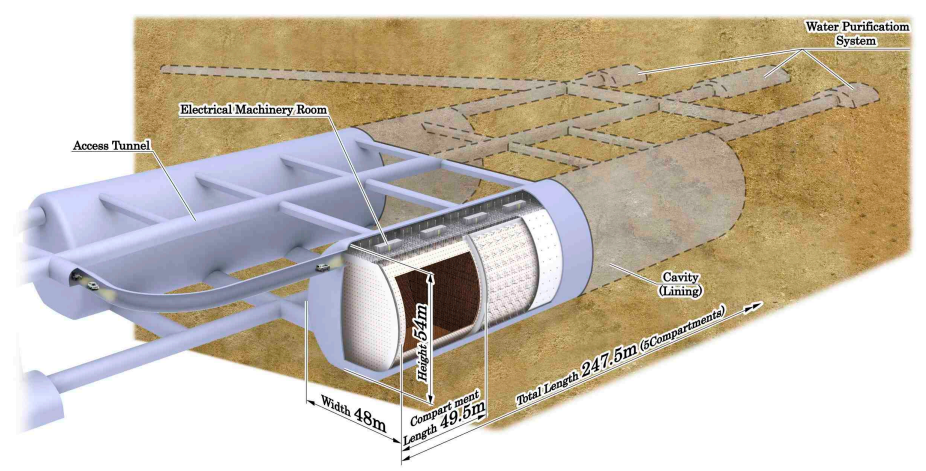

Figure 1: Schematic view of the Hyper-Kamiokande detector.

\subsection{Photo-sensor for Hyper-Kamiokande}

The base design of Hyper-Kamiokande utilizes the same photo-sensor as Super-Kamiokande, which has well established performance and stability. The Hyper-K tank is optically separated into inner detector and outer detector regions to detect neutrinos in the inner volume with vetoing cosmic-ray muons detected in the outer volume (Figure 『). The inner detector will have 99,000 20inch PMTs which is nine times as many as Super-Kamiokande. A comparison of the two detectors appears in Table $\mathrm{C}$. In addition to a larger volume at Hyper-Kamiokande an upgraded photo-sensor option is being considered.

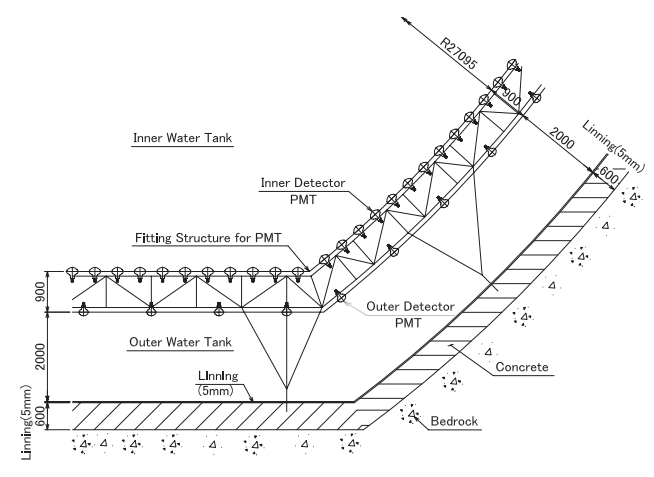

Figure 2: Cross section (lower corner) of Hyper-Kamiokande.

One candidate photo-sensor is the Hybrid Photo-detector (HPD), which is described in the following sections (Section Q, [B]). Because HPDs are yet to be used in a large water Cherenkov detector, a proof-of-principle test using a 200-ton water tank is planned (Section $⿴$ ). 
Table 1: Number and photo-coverage comparison. Although the same quantum efficiency (Q.E.) as the Super-K PMTs is assumed for Hyper-Kamiokande, there is an option to upgrade the Q.E. to $30 \%$ level

\begin{tabular}{lrr}
\hline \hline & Hyper-Kamiokande & Super-Kamiokande \\
\hline Inner detector & $99,000($ 20-inch PMT) & $11,129(20$-inch PMT) \\
Outer detector & $25,000(8$-inch PMT) & $1,885(8$-inch PMT) \\
Photo-coverage & $20 \%$ & $40 \%$ \\
Quantum efficiency & about 30\% & $22 \%$ \\
\hline \hline
\end{tabular}

\section{Hybrid Photo-detector}

The Hybrid Photo-detector is a device that combines a phototube and an electron detector, which can play the role of a typical PMT. For a large water Cherenkov detector such as Hyper-K, a large HPD with an avalanche diode (AD) is being developed.

Figure B 3 shows a schematic view of the (a) PMT and (b) HPD detection principles. Though the phototube part as well as the photocathode is common between PMTs and HPDs, the HPD requires a higher operating voltage, around $8 \mathrm{kV}$, compared to the $1-2 \mathrm{kV}$ of a standard PMT. For the HPD this higher electron-bombardment gain is desired because of the low gain of the AD around 100 : the product of these two is the total gain of the HPD. An $8 \mathrm{kV}$ bias gives a multiplication factor of 500 , which can be compared to the factor of 5 electron-bombardment gain found in PMTs. Another reason to apply the high voltage is to focus photo-electrons onto the small diameter of the AD. This focusing is to within $5 \mathrm{~mm} \phi$ at $\mathrm{AD}$ for the 8-inch HPD.

A large bias voltage induces a fast electron drift off of the photocathode and since the drift length in the AD is uniform compared to a PMT's dynode, the timing performance of the HPD is expected to be superior. In addition it should have a higher tolerance to magnetic fields. The high electron-bombardment gain at the first step gives the benefit of a good signal resolution with less noise at the HPD and single photo-electrons can be clearly seen. In addition, good gain uniformity is expected in the HPD thanks to the axial symmetry of AD. The production cost of the HPD is expected to be lower than that of PMTs because the structure of AD is simple and mass production is possible.

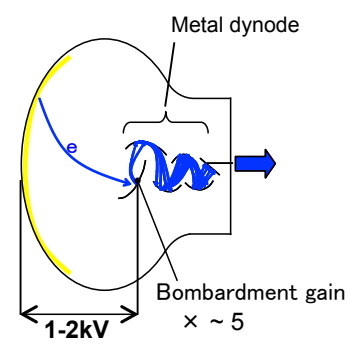

(a) Photomultiplier tube (PMT)

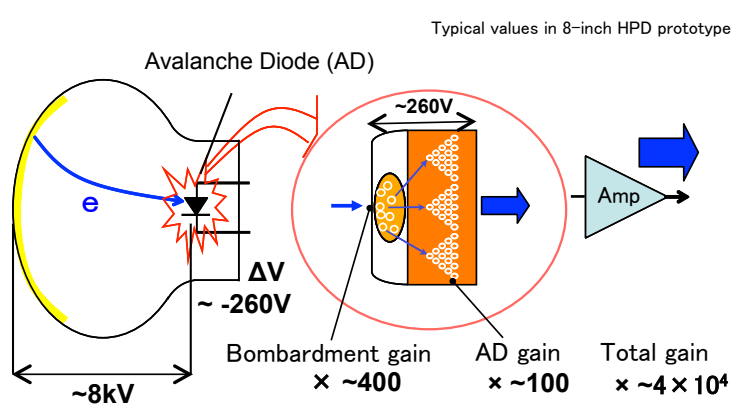

(b) Hybrid Photo-detector (HPD)

Figure 3: Schematic view of PMT and HPD 
Some concerns about the HPD are the requisite high voltage operation and the lack of experience using them in water. Our purpose is to establish their practical use in this type of environment and demonstrate their performance by a proof-of-principle test.

\section{HPD for the proof-of-principle test}

\subsection{8-inch HPD design}

For the first tests in water, an 8-inch diameter HPD has been developed by Hamamatsu Photonics K.K. This HPD is also a candidate for the photo-sensor used in the outer detector of Hyper-K. Based on this experience, a larger 20-inch HPD will be developed within a few years. Table $\square$ lists the specifications of the 8-inch HPD.

Table 2: Specification of 8-inch HPD provided by Hamamatsu Photonics K. K.

\begin{tabular}{lc}
\hline \hline Range of sensitive wavelengths & $300-650 \mathrm{~nm}$ \\
Photocathode bias voltage & $8 \mathrm{kV}$ \\
Avalanche diode bias voltage & $260 \mathrm{~V}$ \\
HPD gain & $4 \times 10^{4}$ \\
Single-photon resolution $(\sigma)$ & $20 \%$ \\
Transit time spread (T.T.S.) $(\sigma)$ & $620 \mathrm{ps}$ \\
\hline \hline
\end{tabular}

The HPD has a $5 \mathrm{~mm}$ diameter $\mathrm{AD}$ at its center and is equipped with a high voltage power module and a pre-amplifier at its back as shown in Figure 4 . Charge from the AD is converted to a voltage and multiplied in the pre-amplifier. Both the high voltage power supply and the preamplifier in Figure $\square$ are packed into the space of the HPD and operated by an external $10 \mathrm{~V}$ input. The HPD generates a signal similar to a PMT's waveform for a simultaneous data taken with PMTs in the test. There is no high voltage line in the water thanks to low voltage lines to control high voltage power module, which allows for safe and easy operation.

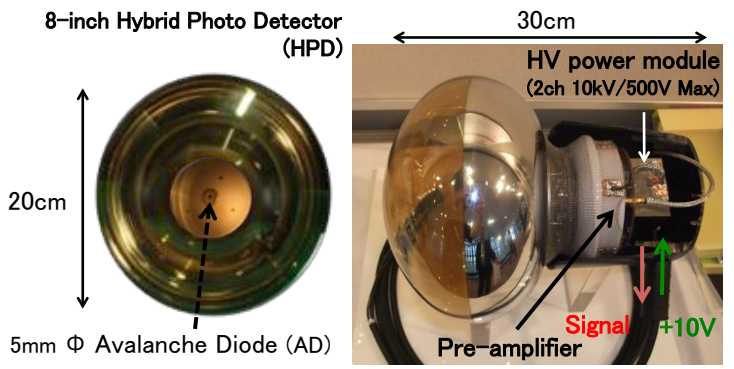

Figure 4: 8-inch HPD and end-cap electronics module

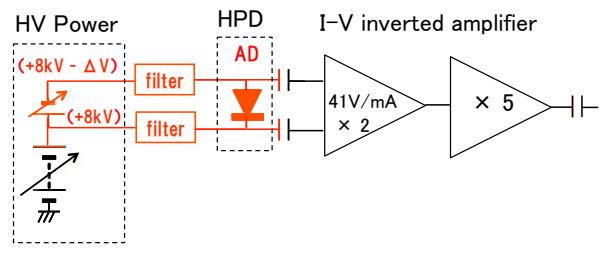

Figure 5: HV and pre-amplifier circuit inside HPD

\subsection{Performance evaluation of the HPD}

Though the exact specifications of the HPD for this test is still under development, a prototype 8 -inch HPD is being studied to evaluate its performance. Figures 6 and $\square$ show clear separation 
of the 1-, 2-, and 3- photo-electron peaks in the HPD waveform and their charge distribution, respectively. The peak-to-valley ratio, which is calculated using the peak height at 1 photo-electron and the valley between the pedestal and this peak, is a good parameter to indicate trigger performance. This measured value for the prototype HPD is 6.0. Though this is excellent compared to the 1.9 peak-to-valley ratio for a 20-inch PMT, it might change in the future because it depends on the measurement setup, such as a temperature, a charge integration time and a pulse height taken instead of charge.

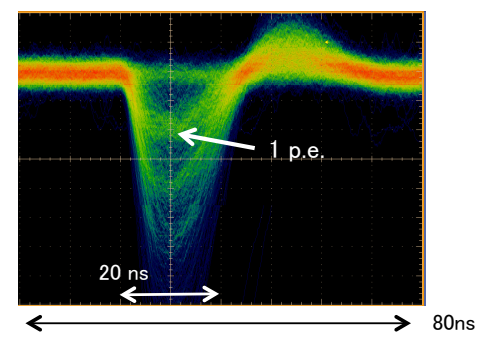

Figure 6: Waveform from HPD output.

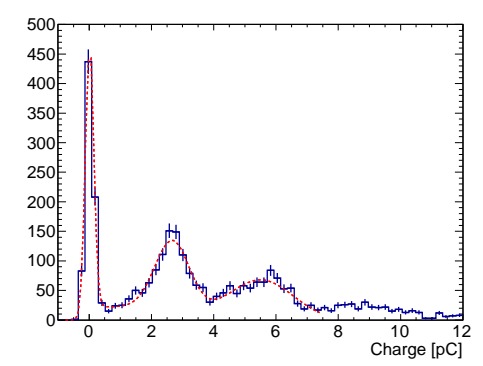

Figure 7: Photo-electron charge distribution.

\subsection{Preparation for the proof-of-principle test}

Before introducing HPDs into the 200-ton water tank, several measurements and preparations are required to confirm their safe and stable operation in water. A safety test where the $8 \mathrm{kV}$ high voltage is applied while the HPD is in water is planned to confirm there is no discharge outside HPD. Also durability is checked to avoid a breakdown of the electronics inside the HPD by operating the HPD for a long time. Aging is simulating by exposing the HPD to a large amount of light and by frequently switching high voltage values. The first long test of the HPD lasted for two days and good stability of the gain was observed (Figure $\mathbb{8}$ ). The gain of the 8-inch HPDs will be calibrated using avalanche bias voltages and their performance will be measured in advance of the full in-situ test.
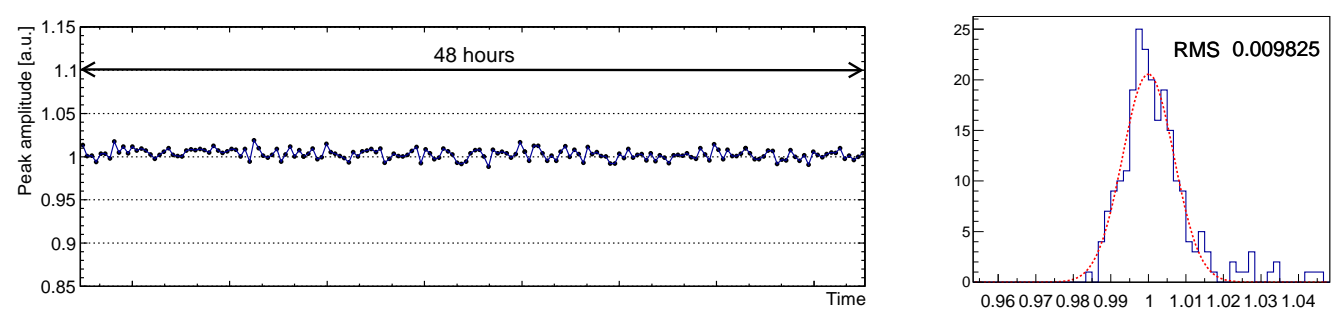

Figure 8: HPD gain stability for 48 hours and its projection.

\section{Proof-of-principle test of HPD in 200-ton water tank}

The purpose of the HPD testing is to evaluate their performance within a water Cherenkov detector and to confirm their long term stability. Measuring their time and charge resolution could additionally give the expected performance of Hyper-Kamiokande with HPDs. 
There is a plan to install eight 8-inch HPDs into a 200-ton water tank that is being prepared for the EGADS (Evaluating Gadolinium's Action on Detector Systems) experiment. EGADS is an evaluation experiment of the GADZOOKS! (Gadolinium Anti-neutrino Detector Zealously Outperforming Old Kamiokande Super!) proposal [B]], which is designed to tag electron anti-neutrinos using an $8 \mathrm{MeV}$ gamma ray resulting from neutron capture on Gd. Therefore $0.2 \%$ Gadolinium $\left(\mathrm{Gd}_{2}\left(\mathrm{SO}_{4}\right)_{3}\right)$ will eventually be loaded into the EGADS water.

The tank is designed to observe Cherenkov light using 240 20-inch PMTs identical to those of Super-Kamiokande, which allows a relative performance measurement between the HPD and PMT. It has already been constructed and eight 8-inch HPDs will be installed in place of 20-inch PMTs as seen in Figure 8 .
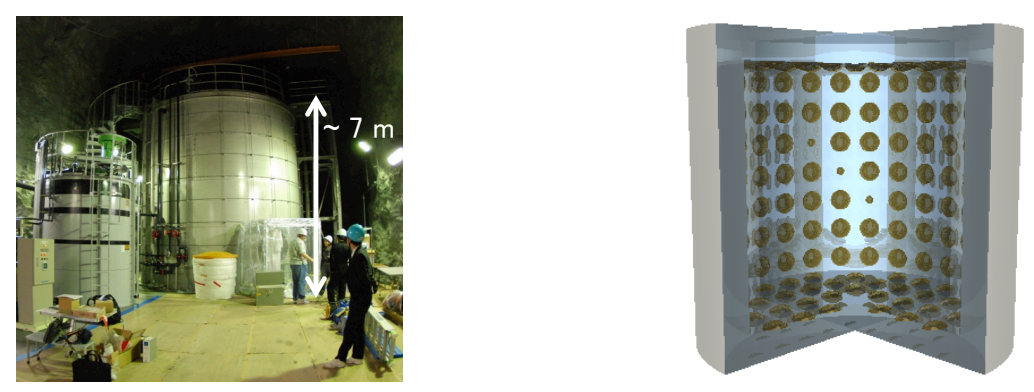

Figure 9: Outside / inside view of EGADS tank, where several HPDs are placed instead of 20-inch PMTs.

The proof-of-principle test will start at the beginning of 2013 and measure the performance of the 8-inch HPDs over the course of a year. The development of the 20-inch HPD is ongoing and a prototype will be mounted in the tank in 2014. This HPD testing will end by 2016 and be used to determine which photo-sensor should be used in for Hyper-Kamiokande.

\section{Summary}

The first proof-of-principle test of the HPD in a 200-ton water tank is planned at the Kamioka mine in Japan thanks to cooperation from Hamamatsu Photonics K.K. and the EGADS collaboration. It will start at the beginning of 2013 and aims to determine the Hyper-K photo-sensor within a few years. Development and performance evaluation activities for the installation of HPD in the tank are now underway.

\section{References}

[1] K. Abe et al., "Letter of Intent: The Hyper-Kamiokande Experiment — Detector Design and Physics Potential —," arXiv:1109.3262 [hep-ex] (2011)

[2] The Super-Kamiokande Collaboration, “The Super-Kamiokande Detector," Nucl. Instrum. Meth. A501 418-462 (2003)

[3] Beacom, John F. and Vagins, Mark R., "Antineutrino Spectroscopy with Large Water Čerenkov Detectors,” Phys. Rev. Lett. 93, 171101 (2004) 